Reprinted from Journal of the Optical Society of America, Vol. 43, No. 3, 218-219, March, 1953

Printed in U. S. A.

\title{
Determination of Absolute $f$ Values from Relative Intensity Measurements for Spectral Lines with Doppler Contour*
}

\author{
S. S. Penner \\ Guggenheim Jet Propulsion Center, California Institute of Technology, \\ Pasadena, California \\ (Received September 11, 1952)
}

$\mathrm{T}$

HE experimental determination of absolute intensities for isolated spectral lines or for entire vibration-rotation bands involves formidable experimental difficulties. For this reason it is highly desirable to consider the use of techniques which permit the determination of absolute $f$ values from relative intensity measurements performed by the use of a low resolution spectrograph. It is the purpose of this note to call attention to a useful experimental procedure for spectral lines with Doppler contour and for optical densities which are sufficiently large to assure a nonlinear dependence of intensity on optical density. The method is a generalization of a two-path experiment proposed for emission studies on flames. ${ }^{1}$

For spectral lines with Doppler contour it is well known ${ }^{2}$ that the total absorbed intensity of radiation $A\left[\nu_{l u}(K)\right]$, for the line identified by the index $K$ and with center at the frequency $\nu_{l}$, is

$$
\begin{aligned}
& A\left[\nu_{l u}(K)\right] \simeq R^{0}\left(\nu_{l u}\right)\left(m c^{2} / 2 \pi k T \nu_{l u}^{2}\right)^{-\frac{2}{2}}\left[P_{\max }(K) X\right] \\
& \times\left\{\sum_{n=0}^{\infty}\left[(n+1)^{\frac{1}{2}}(n+1) !\right]^{-1}\left[-P_{\max }(K) X\right]^{n}\right\},
\end{aligned}
$$

if $R^{0}\left(\nu_{l u}\right)$ is the intensity of the incident radiation at $\nu_{l u}, m$ is the mass of the absorber, $c$ equals the velocity of light, $k$ is the Boltzmann constant, $T$ represents the absolute temperature, $X$ is the optical density (in cm-atmos) of the absorber, and $P_{\max }$ is the maximum value of the spectral absorption coefficient (in $\mathrm{cm}^{-1}$ atmos ${ }^{-1}$. The quantity $P_{\max }$ is related to the integrated intensity $S$ (in $\mathrm{cm}^{-2}$ atmos ${ }^{-1}$ ) through the relation

$$
P_{\max }=S\left(m c^{2} / 2 \pi k T_{\nu_{l u}}\right)^{2} \text {; }
$$

the quantity $S$ (in $\mathrm{cm}^{-2}$-atmos ${ }^{-1}$ ) is related to the dimensionless $f$ value through the expression

$$
S=2.3789 \times 10^{7}(273.1 / T) f \text {. }
$$

It is apparent from Eq. (1) that the dimensionless ratio $R=A\left[\nu_{l u}(K)\right] / R^{0}\left(\nu_{l u}\right)\left(m c^{2} / 2 \pi k T \nu_{l u}{ }^{2}\right)^{-t}$ is a unique function ${ }^{2}$ of $P_{\max } X$, i.e., $R=\varphi\left(P_{\max } X\right)$. If $R=R_{1}$ for $X=X_{1}$ and $R=R_{2}$ for $X=X_{2}$, then $R_{1} / R_{2}=\varphi\left(P_{\max } X_{1}\right) / \varphi\left(P_{\max } X_{2}\right)=\varphi^{1}\left(P_{\max } X_{1}, X_{1} / X_{2}\right)$. The function $\varphi^{1}$ can be determined without difficulty for arbitrary values of $X_{1} / X_{2}$. For $X_{1} / X_{2}=2$ the results have been published elsewhere. ${ }^{1}$ From the known values of $X_{1} / X_{2}$ and the measured values of $R_{1} / R_{2}$ it is a simple matter to obtain $P_{\max } X_{1}$ and hence $P_{\max }$ or $S$. In this manner absolute $f$ values can be determined from relative intensity measurements for spectral lines with Doppler contour.

For studies in the visible and ultraviolet regions of molecular spectra, and at ordinary temperatures, $h \nu_{l u} \gg k T$ and ${ }^{1}$

$$
\frac{\partial \ln \left\{P_{\max }(K) X /\left[g_{u}\left(q_{l_{u}}\right)^{2}\right] K\right\}}{\partial\left(E_{u}-h \nu_{l u}\right)}=-1 / k T,
$$

where $g_{u}\left(q_{L_{u}}\right)^{2}$ represents the relative transition probability for the line with index $K, E_{u}$ is the energy of the upper state, and $h$ is Planck's constant. At fixed values of $T$, Eq. (2) is useful for checking the consistency of experimentally determined values of $P_{\max }(K) X$.

The two-path absorption experiments can be generalized to spectral lines with combined Doppler- and collision-broadening if the line shape is known or is to be measured.

* Supported by the U. S. Office of Naval Research under Contract Nonr$220(03)$, NR 015210 .

I S. S. Penner, J. Chem. Phys. 20, 1341 (1952)

2 R. Ladenburg, $Z$. Physik 65, 200 (1930). 